\title{
Characteristics and outcomes of patients after multiple $(\geq 3)$ catheter ablation procedures for atrial fibrillation
}

\section{Hao Wang}

Shanghai Jiao Tong University Affiliated Chest Hospital Jindong Chen

Shanghai Jiao Tong University Affiliated Chest Hospital

Mengmeng Zhou

Shanghai Jiao Tong University Affiliated Chest Hospital

Liang Zhao ( $\square$ zhaoliang80112@126.com )

Shanghai Jiao Tong University Affiliated Chest Hospital https://orcid.org/0000-0001-8209-559X

Research article

Keywords: atrial fibrillation; multiple catheter ablation procedures; conversion of recurrent types;

pulmonary vein potential

Posted Date: August 6th, 2019

DOI: https://doi.org/10.21203/rs.2.12472/v1

License: (c) (i) This work is licensed under a Creative Commons Attribution 4.0 International License.

Read Full License 


\section{Abstract}

Background: Many atrial fibrillation (AF) patients require more than one radiofrequency catheter ablation (RFCA) procedure to maintain sinus rhythm. This study aimed to evaluate risk and risk factors of atrial tachyarrhythmia (ATa) recurrence in patients undergoing multiple $(\geq 3)$ RFCA procedures for AF. Methods: This single-center, retrospective, observational study enrolled 118 consecutive patients who underwent multiple ablation procedures for paroxysmal and non-paroxysmal AF with circumferential pulmonary vein ablation (CPVA), and bidirectional block of lines with disappearance of complex fractionated atrial electrograms (CFAEs) as index procedural endpoints, respectively. Results: At a median follow-up of 18 (range, 6-91) months after the last procedure (mean, 3.2 procedures), freedom from ATa recurrence was 40.7\% (48/118). Initially diagnosed non-paroxysmal AF ( $P=0.039)$, baseline LA size $(P=0.044)$, and recurrent $A F$ after the second procedure $(P=0.044)$ were univariate predictors of $A T$ a recurrence, while only the latter $(\mathrm{P}=0.010)$ was an independent multivariate predictor (hazard ratio for ATa recurrence of 1.88 [95\% Cl, 1.16-3.05]. Conversion of recurrent types between AF and AFL/AT occurred in 52.9\% (37/70) of patients with ATa recurrence, and $29.2 \%(14 / 48)$ of patients with sinus rhythm after last procedure. Few patients (7.8\% [20/257]) recovered PV potential induced recurrent ATa during multiple procedures, and most (87.6\% [141/161]) were bystanders of recurrent ATa. Conclusions: Multiple (>3) RFCA for paroxysmal or non-paroxysmal AF yielded unsatisfactory ATa recurrence rates with recurrent AF after the second procedure as multivariate predictor and recovered PV potential as a bystander commonly as underlying mechanism. Conversion of recurrent types between AF and AFL/AT was common.

\section{Background}

Radiofrequency catheter ablation (RFCA) for atrial fibrillation (AF) has been proved more effective in maintaining sinus rhythm (SR) than antiarrhythmic drugs in experienced centers with adequately trained teams. ${ }^{[1]}$ After single procedure, SR maintenance could be achieved in up to $70 \%$ for paroxysmal AF patients and $50 \%$ for persistent AF patients without symptomatic recurrences. ${ }^{[1]}$ However, it was reported that long-term outcome after single procedure was unsatisfactory, ${ }^{[2,3]}$ and a substantial amount of patients required more than one procedure to obtain symptom control. ${ }^{[1]}$ Although the success rate improves significantly after multiple procedures, ${ }^{[4]}$ atrial tachyarrhythmia (ATa) recurrence remains a limitation. The present study was performed to assess ATa recurrence and its risk factors in patients undergoing multiple $(\geq 3)$ ablation procedures for paroxysmal or non-paroxysmal AF.

\section{Methods}

\subsection{Patients}

The present study retrospectively enrolled 118 consecutive patients with multiple $(\geq 3)$ catheter ablation procedures at our institution. Standard Doppler transthoracic echocardiography was performed for every patient before the procedure. Transesophageal echocardiography was performed to exclude left atrial (LA) thrombi. Definitions of paroxysmal, persistent, and longstanding persistent AF (LSP-AF) conformed 
to the ESC guidelines. ${ }^{[1]}$ Patients were on long-term anticoagulation with therapeutic warfarin and bridging low molecular weight heparin was used during the ablation procedure. Except for amiodarone, other antiarrhythmic drugs were discontinued $\geq 5$ half-lives before the procedure.

\subsection{Catheter ablation}

\subsubsection{Index procedure}

The electrophysiological study (EPS) details have been described in our previous work. ${ }^{[5]}$ For patients with paroxysmal AF, circumferential pulmonary vein ablation (CPVA) was performed and pulmonary vein isolation (PVI) was monitored during the procedure and subsequently confirmed with a circular mapping catheter in each PV. In patients with persistent and LSP-AF, the first ablation step was CPVA to obtain PVI. Secondly, three lines were ablated as follows: 1 ) the roof line, between the two PV circles; 2) the mitral isthmus (MI) line, between the mitral annulus and the left inferior pulmonary vein; and 3 ) the right atrial cavotricuspid isthmus (CTI) line, if atrial tachycardia was consistent with CTI-dependent atrial flutter. Thirdly, complex fractionated atrial electrograms (CFAEs) were mapped and ablated in LA. CFAEs were defined as: 1) atrial fractionated electrograms composed of two deflections or more, and/or perturbation of the baseline with continuous deflection of a prolonged activation complex over a 10-second recording period; and 2) atrial electrograms with a very short cycle length $(<120 \mathrm{~ms})$ averaged over a 10 -second recording period. ${ }^{[6]}$ On the endocardium, irrigated radiofrequency energy was delivered during CPVA with a flow rate of $20 \mathrm{~mL} / \mathrm{min}$ and a maximum power of $30 \mathrm{~W}$, and during CFAEs and linear ablation with a flow rate of $25 \mathrm{~mL} / \mathrm{min}$ and a maximum power of $35 \mathrm{~W}$. Flow and power of ablation were limited to an irrigation rate of $25 \mathrm{~mL} / \mathrm{min}$ and a maximum power of $25 \mathrm{~W}$ inside the coronary sinus (CS). If AF termination was not achieved after the latter steps, cardioversion was used to restore SR. Under SR, PVI was reconfirmed and additional linear ablation undertaken if necessary to obtain bidirectional block of lines.

\subsubsection{Repeat procedures}

During repeat procedures, if the patient presented with atrial flutter/atrial tachycardia (AFL/AT), activation and entrainment mapping were used to identify underlying mechanisms and to guide the ensuing ablation. Burst atrial pacing or infusion of isoproterenol was used to induce AF in patients with recurrent paroxysmal AF. For patients of initially diagnosed paroxysmal AF with recurrent AF, firstly, PVI was reconfirmed with a circular mapping catheter at each PV, and CPVA was used to eliminate recovery of PV potential. Secondly, common non-PV triggers (eg. superior vena cava, CS, and terminal crest) were mapped and ablated according to procedural findings. Thirdly, CFAEs were mapped and ablated in the LA and the right atrium (RA) and three lines were ablated. For patients with initially diagnosed nonparoxysmal AF with recurrent $A F$, application of CPVA and reconfirmation of PVI were performed as mentioned above; then, common non-PV triggers (eg. superior vena cava, CS, and terminal crest) were mapped and ablated according to procedural findings. Thirdly, CFAEs were remapped and ablated in the 
LA and the RA. Fourthly, after restoring SR by ablation or cardioversion, bidirectional block of three lines was reconfirmed (supplemental file).

\subsection{Follow-up}

All patients were assessed during follow-up using 12-lead electrocardiography (ECG); 24-hour ambulatory ECG monitoring and transthoracic echocardiography. All patients were anticoagulated with warfarin with a target international normalized ratio of 2 to 3 or with new oral anticoagulant drugs. After the procedure, in all cases, treatment with antiarrhythmic drugs was continued for 3 months, and during this blanking period arrhythmia recurrence was managed medically (propafenone, amiodarone, or metoprolol). Patients were instructed to obtain an additional ECG every time they had symptoms. 24-hour Holter monitoring was scheduled at 1, 3, 6, 9 and 12 months, and thereafter every 6 months. Drug-free AF survival was the main outcome after a 3-month blanking period.

\subsection{Statistical analysis}

Normally distributed continuous variables were expressed as mean \pm standard deviation and were compared using independent-samples t-test or one-way ANOVA. Non-normally distributed continuous variables were expressed as median (interquartile range) and were compared using Mann-Whitney-U test. Categorical variables were expressed as numbers and percentages and were compared using $\chi^{2}$ test. Event-free survival was estimated by the Kaplan-Meier method and compared using the log-rank test. Univariate and multivariable Cox regression models were used to assess the association between baseline patient characteristics and the risk of ATa recurrence. Each patient baseline characteristic was examined by univariate analysis with the study outcome denoted as the dependent variable and baseline patient characteristic as the covariate. Significant covariates $(P<0.05)$ identified from the univariate analyses were then included in a multivariable Cox regression model. Covariates that were significant in the multivariable model $(P<0.05)$ were considered independently associated with the study outcome. Hazard ratios and the corresponding 95\% confidence intervals were estimated with the use of Cox proportional-hazards models. A two-tailed $\mathrm{P}$ value $<0.05$ was considered statistically significant.

\section{Results}

Baseline characteristics of the 118 patients before index ablation are presented in Table 1. Patients studied had either paroxysmal AF (36 [30.5\%]) or non-paroxysmal AF (82 [69.5\%]) for a median of 24 months after diagnosis. The mean age was $60 \pm 9$ years; the mean LA diameter was $44.1 \pm 6.3 \mathrm{~mm}$. RA enlargement, Mitral regurgitation and tricuspid regurgitation were observed in 52 (44.1\%) patients, 67 (56.8\%) patients and $35(29.7 \%)$ patients, respectively.

\subsection{Procedural outcomes}

\subsubsection{Index procedure}


In paroxysmal AF patients, CPVA and PVI was achieved in all patients, with additional LA CFAEs ablation performed in 3 patients when AF occurred during the procedure (2 AF terminated, and $1 \mathrm{AF}$ converted to AFL). Among the 82 non-paroxysmal AF patients, SR restoration was achieved during ablation procedure in 8 patients (during CFAEs ablation at CS in 2 patients, LA front wall in 1 patient, and roof in 1 patient; during $\mathrm{CTI}$ ablation in 2 patients and $\mathrm{MI}$ ablation in 2 patients), and cardioversion was applied to restore SR in the remaining 74 patients.

\subsubsection{Second procedure}

During the second procedure, in 42 patients with AFL/AT, 36 AFL/ATs were identified including macroreentry in 26 patients (7 Ml-related ATs, 5 CTI-related ATs, 4 roof-related ATs, 3 LA front-wall-related ATs, and 7 multiple-reentries-related ATs), microreentry or foci AFL/AT in 5 patients (2 LA front-wall-related ATs, 1 LA-appendage-related AT, 1 atrial-septum-related AT, and 1 mitral-annulus-related AT) and PV triggers in 5 patients. The mechanisms of the remaining 6 AFL/ATs were unclear due to inconsistent entrainment results, frequent transition to other AT during entrainment, or unexplainable activation mapping results.

Among the 76 patients with recurrent AF, non-PV triggers were observed in 11 patients ( 5 from superior vena cava, 3 from CS, 1 from terminal crest, 1 from left atrial appendage and 1 from LA roof). PV reconnection was observed in 93 (78.8\%)atients during the second procedure including 67 with recurrent AF and 26 with recurrent AFL/AT after the first ablation, and induced AF or AFL/AT in 14 (15.1\%) patients, with the rest 79 recurrent ATa (84.9\%) unassociated with PV potential. Complete PVI was obtained in 25 $(21.8 \%)$ patients during the second procedure, including 9 with recurrent $A F$ and 16 with recurrent AFL/AT after the first ablation.

Recovered conduction of lines was observed in 18 of 82 non-paroxysmal AF patients (22.0\%) undergoing linear ablation during index procedure.

\subsubsection{Third procedure}

After the second procedure, among the 42 patients with recurrent AFL/AT, 34 had AFL/AT recurrence and the other 8 recurred with AF; among 76 paitents with recurrent AF, AF recurred in 46 patients and AFL/AT recurred in 30 patients after the second procedure. During the third procedure, among 64 AFL/ATs, mechanisms of $54 \mathrm{AFL} / \mathrm{AT}$ were identified including macroreentry in 46 patients ( $20 \mathrm{Ml}$-related, $5 \mathrm{CTI}$ related, 5 roof-related, 4 LA front wall-related, and 12 multiple reentries-related ATs), microreentry/foci in 6 patients (2 LA front-wall-related, 1 roof-related, 1 CS-related, and 2 mitral annulus-related ATs) and PV triggers in 2 patients. The mechanisms remained unkown in the other 10 AFL/ATs. In the 54 patients with recurrent AF, non-PV triggers were found in 9 patients (3 superior vena cava, 1 CS, 3 LA anterior wall, 1 LA appendage, and $1 \mathrm{LA}$ roof).

During the third procedure, PV reconnection was observed in 66 (55.9\%) patients including 31 with recurrent AFL/AT and 35 with recurrent AF after the second ablation. Recovered PV potential was 
discovered to associate with $6(9.1 \%)$ patients and acted as bystander in the other 60 patients $(90.9 \%)$. Recovered conduction of lines was observed in 30 of 83 patients who had undergone linear ablation during first and second procedure. Patients with recurrent $\mathrm{AF}$ after the second procedure had longer $\mathrm{AF}$ duration than those with AFL/AT $(P=0.036)$.

\subsubsection{Over three procedures}

After the third procedure, 25 of 64 (39.1\%) recurrent AFL/AT patients and 17 of 54 (31.5\%) recurrent AF patients remained in SR. And 17 of 76 patients with recurrent ATa underwent the fourth ablation including $12 \mathrm{AFL} / \mathrm{AT}$ and $5 \mathrm{AF}$. The mechanisms of $10 \mathrm{AFL} / \mathrm{AT}$ were identified including 10 macro-reentry (2 MIrelated, 1 CTI-related, 1 RA-related, and 6 multiple reentries-related ATs) and the mechanisms of the other 2 remained unclear. During the fourth EPS, complete PVI was observed in 15 patients including 11 with $\mathrm{AFL} / \mathrm{AT}$ and 4 with $\mathrm{AF}$, and PV reconnection was observed in the other $1 \mathrm{AF}$ and $1 \mathrm{AFL} / \mathrm{AT}$ but unassociated with ATa recurrence.

After the fourth procedure, 4 of $12 \mathrm{AFL} / \mathrm{AT}$ patients remained in SR and another $4 \mathrm{AFL} / \mathrm{AT}$ patients underwent the fifth procedure, among which 2 patients remained in SR, while no AF patients remained in SR or underwent fifth ablation. Complete PVI was observed in all 4 patients during the fifth EPS, and multiple reentries-related macroreentry ( 2 LA roof-MI-related ATs, and 1 CS-MI-related ATs) was identified in 3 patients other 1 remained unclear. Two patients remained in SR after the fifth procedure.

\subsubsection{ATa recurrence types}

Among the 70 patients with ATa recurrence after the last procedure, AF recurrence was observed in 22 (31.4\%) patients, AFL/AT in 11 (15.7\%), and conversion between AFL/AT and AF in the other 37 (52.9\%). Among the 48 patients remaining in SR after the last procedure, 16 (33.3\%) underwent multiple recurrent AF, 18 (37.5\%) underwent multiple recurrent AFL/AT, and the other 14 (29.2\%) underwent conversion between AFL/AT and AF. Conversion between different recurrent types was common among patients with multiple ATa recurrences. After index and second procedures, an increasing trend was observed in postablation AFL/AT (from 35.5\% to 54.2\%), including multiple reentries-related macroreentry AFL/AT (from $17.1 \%$ to $18 \%$ ) and AFL/AT with unidentified mechanisms (from $14.3 \%$ to $15.6 \%$ ). By contrast, a decreasing trend was observed in PV-related AFL/AT (from 11.9\% to 3.1\%). After index procedure, PV reconnection was associated with few ATa recurrences (15.1\%), and a potential decreasing trend was observed (from $15.1 \%$ to $9.1 \%$ ) after the second procedure. The types of recurrence after multiple procedures are shown in Figure 1.

\subsection{Follow-up}

After the last procedure with a median follow-up of 18 months (range, 6-91 months), 48 of 118 (40.7\%) patients remained SR after mean 3.2 procedures (Figure 2A), including 21 of 36 (58.3\%) paroxysmal AF patients and 27 of $82(32.9 \%)$ non-paroxysmal AF patients ( $P=0.010$, for comparison). Recurrent AF after the second procedure was associated with a higher risk for ATa recurrence than AFL/AT (Figure 2B). After 
multiple procedures, no significant difference was observed between patients with and without complete PVI confirmed by the third EPS (Figure $2 \mathrm{C}$ ). In univariate Cox regression model, initially diagnosed nonparoxysmal AF $(P=0.039)$, larger baseline LA diameter $(P=0.044)$, and recurrent AF after the second procedure were associated with a higher risk of ATa recurrence. And in multivariate Cox regression model, only recurrent $A F$ after the second procedure was an independent predictor of $A T a$ recurrence $(H R=1.88$, 95\% Cl [1.16-3.05]; $P=0.010$, Table 2).

\section{Discussion}

The present study documenting ATa recurrence and its predictors after multiple ( $\geq 3)$ RFCA procedures for paroxysmal or non-paroxysmal AF had the following major findings: 1) long-term outcome was unsatisfactory for AF patients with multiple ATa recurrences; 2) recurrent AF after the second procedure was an independent predictor of ATa recurrence, and was associated with a higher recurrent risk; 3 ) conversion between AF and AFL/AT was common in patients with multiple ATa recurrences; and 4) recovered $\mathrm{PV}$ potential acted as bystander rather than the responsible mechanism in most recurrent ATa after multiple procedures.

Previous studies reported rather favorable outcome of multiple $(\geq 3)$ RFCA procedures for AF patients. ${ }^{[7-9]}$ However, our study yielded different results after mean 3.2 procedures with possible reasons as follows. Firstly our study excluded AF patients with one or two RFCA procedures, which constituted the majority of our patients in our institution with rather favorable long-term outcome, so it was probable that our study object had more complex atrial substrate which was prone to recur with ATa. Secondly, all RFCA procedures of every patient were performed in our institution, making it feasible to retrieve as much medical information of each patient as possible, based on which credible speculation could be obtained.

Non-paroxysmal AF, larger LA size, and recurrent AF after the second procedure were found to correlate with ATa recurrence in this unique subgroup of patients. And compared with paroxysmal AF, it is comprehensible that atrial remodeling tends to be severer in non-paroxysmal AF which is usually accompanied with larger LA size.[10] In contrast to AT, which usually presented as organized electrical activity with identifiable mechanisms in most cases, AF represents severer atrial structural remodeling with unclear mechanisms, which facilitates complex reentry or rapid ectopic firing. So in contrast to recurrent $A F L / A T$, recurrent $A F$ after the second ablation indicated higher level of atrial fibrosis with a higher risk of ATa recurrence after the third procedure. On one hand, AF itself is an important promoter of atrial fibrosis.[11] In our study, patients with recurrent AF after the second procedure had a longer AF duration before procedures than those recurring with AFL/AT, thus more likely to possess more extensive atrial fibrosis. On the other hand, extensive ablation-induced lesions after multiple procedures also play an important role, especially when LA substrate modification ablation was performed. ${ }^{[1]}$ While lesions for AFL/AT with identifiable mechanisms are mostly limited to the critical isthmus. Previous studies also reported less favorable outcome of RFCA for patients with recurrent AF than AT, ${ }^{[12,13]}$ which is consistent with our study. 
As more RFCA procedures were performed, a trend of more patients recurring with AFL/AT rather than AF was observed ( $54.2 \%$ vs. $35.6 \%$, $p=0.04$ for comparison). Complex atrial substrate played an substantial role, and linear ablation could be another possible explanation. Although linear block was the desirable ablation endpoint, it could also act as electrophysiologic barrier, facilitating reentry, especially when gap existed. ${ }^{[14]}$ Thus, as procedures with multiple linear ablations were performed, the risk of atrial gaps also could have increased, which partly accounted for the rising trend of multiple reentries-related macroreentry AFL/AT (16.7\% [7 of 42] vs. 18.75\% [12 of 64] identified during the second, third procedure, respectively).

Reported incidence rate of AF after successful AFL ablation is variable, ranging from $18 \%-50 \%{ }^{[15][16]}$ It was reported that prior AF was the predictor of transition to AF after CTI AFL ablation, ${ }^{[17]}$ whereas others suggested that AF could be initiated by AFL itself. ${ }^{[15]}$ In our study, conversion between AF and AFL/AT was common during the process of multiple procedures, indicating that on one hand, PVI plus atrial substrate modification ablation can effectively eliminate AF triggers and maintain AF substrate; on the other hand, successful ablation of AFL might not stop AF progression, ${ }^{[18]}$ therefore AF developed over time after AFL/AT ablation.

Complete PVI is the optimal goal of RFCA. ${ }^{[1]}$ However, $\mathrm{PV}$ reconnection is not uncommon with reported incidence of nearly $80 \%,{ }^{[7,19]}$ and has been widely acknowledged as an important mechanism of recurrent $A F .[20,21,7]$ However, in our study, patients with complete PVI verified during the third procedure had comparable ATa recurrence to those with PV reconnection, during whose EPS non-PV trigger was identified as the underlying mechanism. Non-PV trigger has been described as critical trigger in patients with very late recurrences, ${ }^{[22]}$ and non-PV ectopy initiating $A F$ was reported to be more active as more ablation procedures were performed. ${ }^{[9,8]}$ Besides, non-PV trigger was more frequently observed in recurrent ATa as follow-up progressed. By contrast, recovered PV potential acted as bystander in ATa recurrence rather than underlying mechanism in most recurrence cases, indicating that non-PV trigger deserves more attention in patients with multiple failed ablations, and innovative solution is required for this subgroup of patients.

\section{Limitations}

The present study is limited by its observational and nonrandomized design and its relatively small sample size, with inherent selection bias; its results and conclusions therefore require confirmation in larger randomized controlled trials. Since ATa recurrence was assessed with 24-h Holter monitoring but not with implantable loop recorders or 7-day Holter monitoring before ablation or during follow-up, overall success rate might have been overestimated, particularly in patients with significant AF regression and symptom improvement. Besides, the time span of patient recruitment in this retrospective study was relatively long, improvements in equipment may have influenced the results.

\section{Conclusions}


In AF patients undergoing multiple ( $\geq 3$ ) ablations, the long-term outcome was unsatisfactory, with recurrent $\mathrm{AF}$ after the second procedure as independent risk factor; conversion between AF and AFL/AT was common in patients with multiple ATa recurrences; and recovered PV potential acted as bystander rather than the underlying mechanism in most ATa recurrences.

\section{Abbreviations}

RFCA, radiofrequency catheter ablation

AF, atrial fibrillation

SR, sinus rhythm

ATa, atrial tachyarrhythmia

LA, left atrial

LSP-AF, longstanding persistent atrial fibrillation

EPS, electrophysiological study

CPVA, circumferential pulmonary vein ablation

$\mathrm{PVI}$, pulmonary vein isolation

$\mathrm{MI}$, mitral isthmus

$\mathrm{CTI}$, cavotricuspid isthmus

CFAE, complex fractionated atrial electrogram

$\mathrm{RA}$, right atrial

AFL, atrial flutter

AT, atrial tachycardia

ECG, electrocardiography

\section{Declarations}

Ethics approval and consent to participate

The study was approved by Shanghai Chest Hospital Ethics Committee, and written informed consent requirement was waived. 
Consent for publication

Not applicable.

Availability of data and materials

Data for the present study are not publicly available since additional studies are underway with the same data set.

Competing interests

The authors declare that they have no competing interests.

Funding

This research received no grant from any funding agency in the public, commercial or not-for-profit sectors.

Authors' contributions

HW and JC: data collection, analysis and interpretation, drafting article, and statistics; MZ: data collection, analysis and interpretation; LZ: Concept and design, critical revision of article and approval of article.

Acknowledgements

Not applicable.

\section{References}

1. Kirchhof P, Benussi S, Kotecha D, Ahlsson A, Atar D, Casadei B et al. 2016 ESC Guidelines for the management of atrial fibrillation developed in collaboration with EACTS. Eur Heart J. 2016;37(38):2893-962. doi:10.1093/eurheartj/ehw210. 
2. Ouyang F, Tilz R, Chun J, Schmidt B, Wissner E, Zerm T et al. Long-term results of catheter ablation in paroxysmal atrial fibrillation: lessons from a 5-year follow-up. Circulation. 2010;122(23):2368-77. doi:10.1161/circulationaha.110.946806.

3. Ganesan AN, Shipp NJ, Brooks AG, Kuklik P, Lau DH, Lim HS et al. Long-term outcomes of catheter ablation of atrial fibrillation: a systematic review and meta-analysis. Journal of the American Heart Association. 2013;2(2):e004549. doi:10.1161/jaha.112.004549.

4. Rostock T, Salukhe TV, Steven D, Drewitz I, Hoffmann BA, Bock K et al. Long-term single- and multiple-procedure outcome and predictors of success after catheter ablation for persistent atrial fibrillation. Heart rhythm. 2011;8(9):1391-7. doi:10.1016/j.hrthm.2011.04.012.

5. Zhao L, Xu K, Jiang W, Zhou L, Wang Y, Zhang X et al. Long-term outcomes of catheter ablation of atrial fibrillation in dilated cardiomyopathy. Int J Cardiol. 2015;190:227-32. doi:10.1016/j.ijcard.2015.04.186 S0167-5273(15)00919-5 [pii].

6. Aksu T, Guler TE, Yalin K, Oto A. Unanswered Questions in Complex Fractionated Atrial Electrogram Ablation. Pacing and clinical electrophysiology : PACE. 2016;39(11):1269-78. doi:10.1111/pace.12944.

7. Lo LW, Tai CT, Lin YJ, Chang SL, Wongcharoen W, Hsieh MH et al. Characteristics and outcome in patients receiving multiple (more than two) catheter ablation procedures for paroxysmal atrial fibrillation. Journal of cardiovascular electrophysiology. 2008;19(2):150-6. doi:10.1111/j.15408167.2007.01012.x.

8. Mohanty S, Trivedi C, Gianni C, Della Rocca DG, Morris EH, Burkhardt JD et al. Procedural findings and ablation outcome in patients with atrial fibrillation referred after two or more failed catheter ablations. Journal of cardiovascular electrophysiology. 2017. doi:10.1111/jce.13329.

9. Lo L, Lin Y, Chang S, Hu Y, Chao T, Chung F et al. Predictors and Characteristics of Multiple (More Than 2) Catheter Ablation Procedures for Atrial Fibrillation. J Cardiovasc Electrophysiol. 2015;26(10):1048-56.

10. Scherr D, Khairy P, Miyazaki S, Aurillac-Lavignolle V, Pascale P, Wilton SB et al. Five-year outcome of catheter ablation of persistent atrial fibrillation using termination of atrial fibrillation as a procedural endpoint. Circ Arrhythm Electrophysiol. 2015;8(1):18-24. doi:10.1161/CIRCEP.114.001943.

11. Iwasaki YK, Nishida K, Kato T, Nattel S. Atrial fibrillation pathophysiology: implications for management. Circulation. 2011;124(20):2264-74. doi:10.1161/CIRCULATIONAHA.111.019893.

12. Morady F, Oral H, Chugh A. Diagnosis and ablation of atypical atrial tachycardia and flutter complicating atrial fibrillation ablation. Heart rhythm. 2009;6(8 Suppl):S29-32. doi:10.1016/j.hrthm.2009.02.011.

13. Zhao L, Wu S, Jiang W, Zhou L, Gu J, Wang Y et al. Differential clinical characteristics and prognosis of patients with longstanding persistent atrial fibrillation presenting with recurrent atrial tachycardia versus recurrent atrial fibrillation after first ablation. Journal of cardiovascular electrophysiology. 2014;25(3):259-65. doi:10.1111/jce.12311. 
14. Chae S, Oral H, Good E, Dey S, Wimmer A, Crawford T et al. Atrial tachycardia after circumferential pulmonary vein ablation of atrial fibrillation: mechanistic insights, results of catheter ablation, and risk factors for recurrence. Journal of the American College of Cardiology. 2007;50(18):1781-7. doi:10.1016/j.jacc.2007.07.044.

15. Waldo AL, Feld GK. Inter-relationships of atrial fibrillation and atrial flutter mechanisms and clinical implications. Journal of the American College of Cardiology. 2008;51(8):779-86. doi:10.1016/j.jacc.2007.08.066.

16. Tomson TT, Kapa S, Bala R, Riley MP, Lin D, Epstein AE et al. Risk of stroke and atrial fibrillation after radiofrequency catheter ablation of typical atrial flutter. Heart rhythm. 2012;9(11):1779-84. doi:10.1016/j.hrthm.2012.07.013.

17. Seara JG, Roubin SR, Gude Sampedro F, Barreiro VB, Sande JM, Manero MR et al. Risk of atrial fibrillation, stroke, and death after radiofrequency catheter ablation of typical atrial flutter. Clinical research in cardiology : official journal of the German Cardiac Society. 2014;103(7):543-52. doi:10.1007/s00392-014-0682-6.

18. Luria DM, Hodge DO, Monahan KH, Haroldson JM, Shen WK, Asirvatham SJ et al. Effect of radiofrequency ablation of atrial flutter on the natural history of subsequent atrial arrhythmias. Journal of cardiovascular electrophysiology. 2008;19(11):1145-50. doi:10.1111/j.15408167.2008.01206.x.

19. Ouyang F, Antz M, Ernst S, Hachiya H, Mavrakis H, Deger FT et al. Recovered pulmonary vein conduction as a dominant factor for recurrent atrial tachyarrhythmias after complete circular isolation of the pulmonary veins: lessons from double Lasso technique. Circulation. 2005;111(2):127-35. doi:10.1161/01.cir.0000151289.73085.36.

20. Inada K, Yamane T, Tokutake K, Yokoyama K, Mishima T, Hioki M et al. The role of successful catheter ablation in patients with paroxysmal atrial fibrillation and prolonged sinus pauses: outcome during a 5-year follow-up. Europace : European pacing, arrhythmias, and cardiac electrophysiology : journal of the working groups on cardiac pacing, arrhythmias, and cardiac cellular electrophysiology of the European Society of Cardiology. 2014;16(2):208-13. doi:10.1093/europace/eut159.

21. Verma A, Jiang CY, Betts TR, Chen J, Deisenhofer I, Mantovan R et al. Approaches to catheter ablation for persistent atrial fibrillation. The New England journal of medicine. 2015;372(19):181222. doi:10.1056/NEJMoa1408288.

22. Mainigi SK, Sauer WH, Cooper JM, Dixit S, Gerstenfeld EP, Callans DJ et al. Incidence and predictors of very late recurrence of atrial fibrillation after ablation. Journal of cardiovascular electrophysiology. 2007;18(1):69-74. doi:10.1111/j.1540-8167.2006.00646.x.

\section{Tables}


Table.1 Clinical characteristics of the patients before index procedure

\begin{tabular}{lc}
\hline Variable & $\mathrm{N}=118$ \\
\hline Age, y & $60 \pm 9$ \\
\hline Men & $79(66.9)$ \\
\hline AF duration, months (median, IQR) & $24(12-60)$ \\
\hline AF type & $36(30.5)$ \\
\hline -Paroxysmal & $50(42.4)$ \\
\hline -Persistent & $32(27.1)$ \\
\hline -Long-standing persistent & $68(57.6)$ \\
\hline Hypertension & $20(16.9)$ \\
\hline Diabetes & $7(5.9)$ \\
\hline Stroke & $11(9.3)$ \\
\hline Rheumatic heart disease & $4(3.4)$ \\
\hline Cardiomyopathy & $44 \pm 6$ \\
\hline LA diameter, mm & $60(57-63)$ \\
\hline LV ejection fraction (median, IQR) & $52(44.1)$ \\
\hline RA enlargement & $67(56.8)$ \\
\hline MR & $35(29.7)$ \\
\hline TR & $25(21.1)$ \\
\hline AR & 39.8 \\
\hline
\end{tabular}

Values are expressed as mean \pm SD or $\mathrm{n}(\%)$, unless otherwise indicated. AF indicates atrial fibrillation; IQR, interquartile range; LA, left atrium; LV, left ventricle; RA, right atrium; MR, mitral regurgitation; TR, tricuspid regurgitation and $\mathrm{AR}$, aortic regurgitation.

Table.2 Univariate and multivariate analysis of outcomes

\begin{tabular}{|c|c|c|c|c|c|}
\hline \multirow[t]{2}{*}{ Study outcome } & \multirow[t]{2}{*}{ Baseline characteristics } & \multicolumn{2}{|c|}{ Univariate analysis } & \multicolumn{2}{|c|}{ Multivariable analysis } \\
\hline & & HR (95\% CI) & P Value & HR (95\% CI) & P Value \\
\hline \multirow[t]{3}{*}{ ATa recurrence } & Non-paroxysmal AF & $1.83(1.03-3.25)$ & $\mathrm{P}=0.039$ & $1.65(0.84-3.27)$ & $\mathrm{P}=0.149$ \\
\hline & LA size & $1.04(1.00-1.08)$ & $P=0.044$ & $1.03(0.98-1.08)$ & $P=0.226$ \\
\hline & Recurrent AF & $1.63(1.01-2.63)$ & $\mathrm{P}=0.044$ & $1.88(1.16-3.05)$ & $P=0.010$ \\
\hline
\end{tabular}

ATa, atrial tachyarrhythmia; AF, atrial fibrillation; LA, left atrial.

\section{Figures}




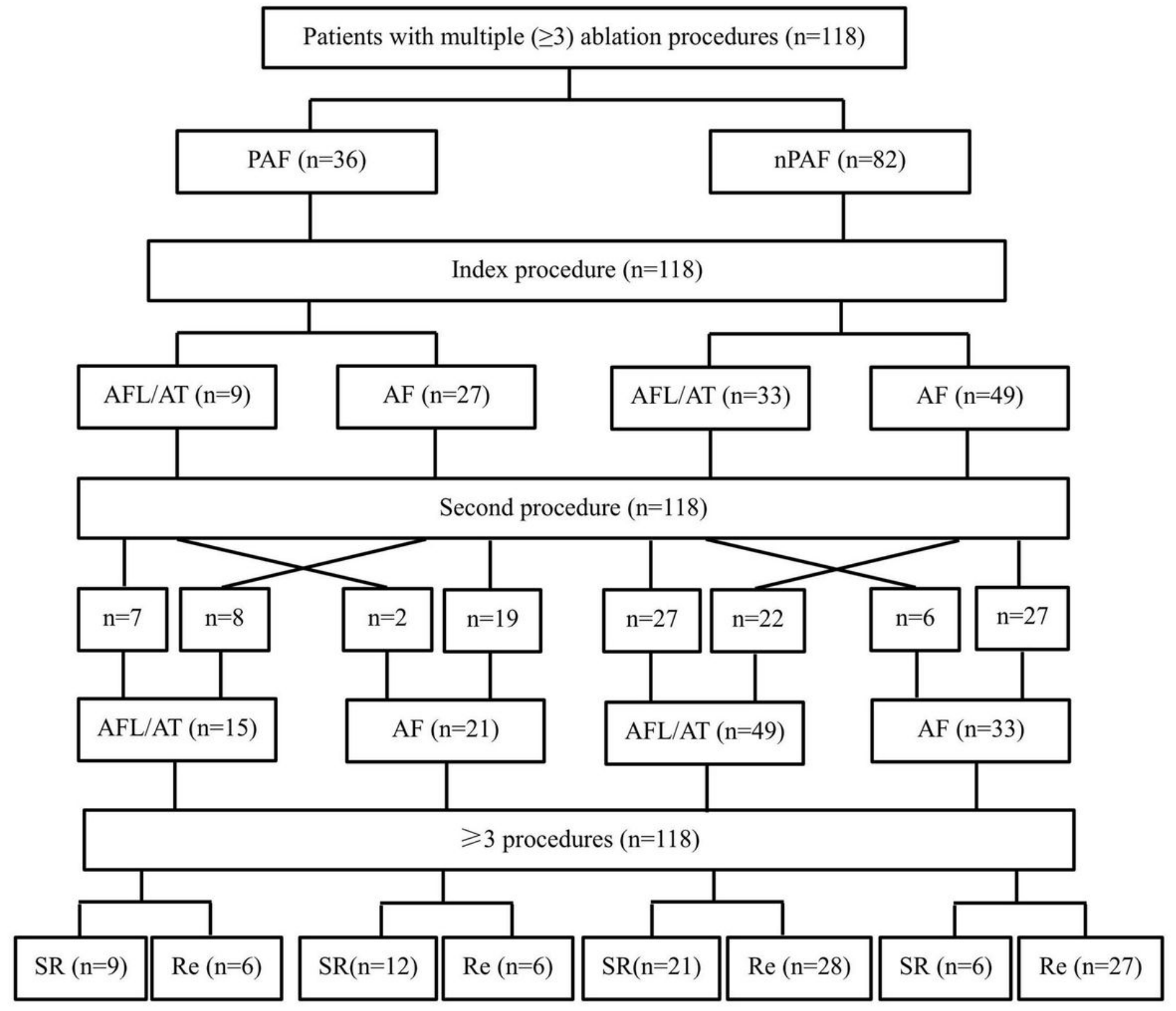

Figure 1

Recurrent types after multiple ablations. AF, atrial fibrillation; AFL, Atrial flutter; AT, atrial tachycardia; PAF, paroxysmal atrial fibrillation; nPAF, non-paroxysmal atrial fibrillation; SR, sinus rhythm; Re, atrial tachycardia recurrence. 

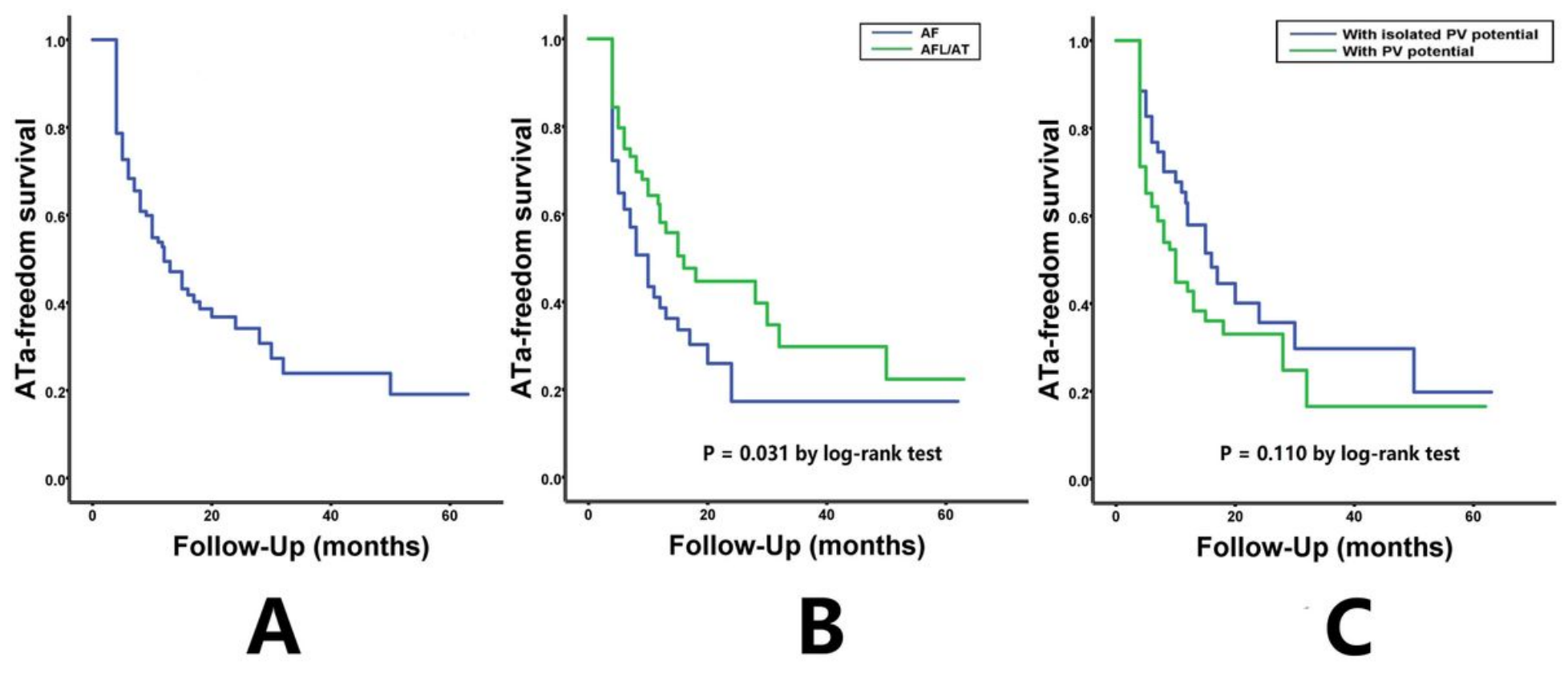

Figure 2

Kaplan-Meier survival curve showing freedom from atrial tachyarrhythmia recurrence for (A) multiple procedures; (B) multiple procedures according to recurrent types; and (C) multiple procedures according to PV potential. ATa indicates atrial tachyarrhythmia; AFL, Atrial flutter; AT, atrial tachycardia; PV, pulmonary vein 